\title{
CLOSED OPERATOR INEQUALITIES AND OPEN PROBLEMS
}

\section{AMEUR SEDDIK}

Abstract. In [3, 4], we have given some characterizations of some subclasses of complex Hilbert space normal operators by inequalities. In this note, we shall reformulate these results using a concept of closed inequalities. Also, we shall give some new characterizations. Some open problems concerning closed inequalities are posed at the end of this note.

Mathematics subject classification (2010): $47 \mathrm{~B} 47$.

Keywords and phrases: Invertible operator, normal operator, unitary operator, self-adjoint operator, unitary reflection operator, operator inequality.

\section{REFERENCES}

[1] G. Corach, R. Porta, And L. Recht, An operator inequality, Linear Algebra Appl., 142 (1990), 153-158.

[2] A. McIntosh, Heinz inequalities and perturbation of spectral families, Macquarie Mathematical Reports, Macquarie Univ., 1979.

[3] A. SEDdiK, Some results related to Corach-Porta-Recht inequality, Proc. Amer. Math. Soc., 129 (2001), 3009-3015.

[4] A. SEDDIK, On the injective norm and characterization of some subclasses of normal operators by inequalities or equalities, J. Math. Anal. Appl., 351 (2009), 277-284. 